\title{
Periocular Recognition using Reduced FEATURES
}

\author{
Ambika D R ${ }^{1}$ Radhika K R ${ }^{1}$ and D Sehsachalam ${ }^{1}$ \\ ${ }^{1}$ BMS College of Engineering, Bangalore - 19, India \\ ambikalieee.org
}

\begin{abstract}
Biometrics is science of measuring and statistically analyzing biological data. Biometric system establishes identity of a person based on unique physical or behavioural characteristic possessed by an individual. Behavioural biometrics measures characteristics which are acquired naturally over time. Physical biometrics measures inherent physical characteristics on a $n$ individual. Over the last few decades enormous attention is drawn towards ocular biometrics. Cues provided by ocular region have led to exploration of newer traits. Feasibility of periocular region as a useful biometric trait has been explored recently. With the promising results of preliminary examination, research towards periocular region is currently gaining lot of prominence. Researchers have analyzed various techniques of feature extraction and classification in the periocular region. The current paper investigates the effect of using Lower Central Periocular Region (LCPR) for identification. The results obtained are comparable with those acquired for the entire periocular region with an advantage of reduced periocular area.
\end{abstract}

\section{KEYWORDS}

Periocular region, Lower Central Periocular Region and ocular biometrics

\section{INTRODUCTION}

Biometric is science of establishing human identification based on physical or behavioural characteristics. An explosive growth is observed in biometric personal authentication and recognition systems for last two decades. Face, iris, ear, palm and finger print are studied extensively. Recent research has explored other human characteristics such as conjunctival vasculature and knuckle joints as supplementary evidence to enhance the performance of classical biometric system.

Ocular biometrics has gained enormous attention after iris pattern recognition evolved. Uniqueness of anatomical architecture of iris and retinal tissues provides ability to achieve user identification. The prime concern of iris is reliable localization under unconstrained environment and acquisition of iris information at large stand-off distances [1].

An improvement of feature extraction methodology and investigation of newer traits is required to address the challenges posed by different orientations of iris images. Periocular region is one such unique trait for recognition purposes, after preliminary exploration of its feasibility $[2,3]$.

\subsection{Periocular Region}

The word periocular is a combination of peri (meaning, the vicinity) and ocular (meaning related to eye). Periocular region refers to skin and anatomical features including birthmarks, eyelids, 
eyelashes contained within a specified region surrounding an eye. Texture of periocular region is reliable over a wide range of distances. The region achieves a good trade-off between iris and face [3].

The periocular features are classified into level-one and level-two features. Level-one features include upper/lower eye folds, upper/lower eyelids, wrinkles and moles as shown in figure 1. Level-two features are more detailed and include skin texture, pores, hair follicles, skin tags and other dermatological features [4].

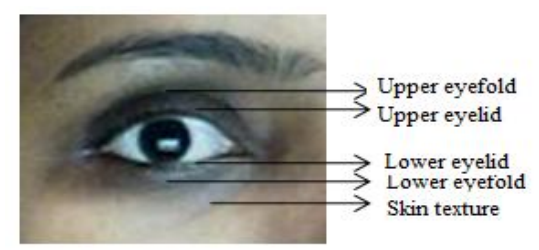

Figure 1. Significant features present in periocular region

\subsection{Challenging concepts of periocular region}

Periocular region provides certain challenges in seeking distinguishing features invariant to transformation in scale, orientation, occlusion, distance and time. Analysis of discriminative powers of features extracted is affected by physical and emotional states of user. The periocular region undergoes deformation in texture and shape due to change in facial expressions, aging, blinking or variations in pose. The noise introduced during acquisition of periocular features is presence of certain artificial elements for instance spectacles, intrusion of hair and eye-lashes. The noise due to these non-optics is termed as occlusion. Information content in periocular region is affected largely by harsh illuminations and other unconstrained conditions.

\section{STATE-OF-THE-ART}

Different regions of periocular area are exhaustively examined in research using various mathematical tools. To eliminate effect of iris texture, co-ordinates of pupil is used to place a circular mask at center of pupil. Further, in some studies an elliptical mask of neutral color is placed in middle of periocular region image to eliminate effect of texture in iris and surrounding sclera area [1, 2 and 9]. Enormous studies are available in literature that has used characteristics of eyebrows in periocular regions along with texture information [2, 10, 11, 12 and 13]. Jameson Merkow, Brendan Jou and Marios Savvides achieve classification rates of $83 \%$ using LBP features in ROI defined by four bounds [8]. Reports from Usang Park, Arun Ross and Anil K Jain using GO, SIFT and LBP indicate up to $81 \%$ accuracy rate without eyebrows and $80 \%$ with the inclusion of eyebrows. Phillip E Miller, Rawls, Pundlik and Woodard experiments have exhibited recognition rates of $85 \%$ using the elliptically masked sclera in periocular region without eyebrows. Fusion of iris and periocular biometrics in Non-ideal imagery is also explored in their studies [14]. Gender classification using periocular region achieve 95\% accuracy through studies of Jamie, Miller, Pundlik and Woodard. Genetic based feature extraction by Adams et al. claims up to $86 \%$ accuracy with reduced features [15]. Samarth Bharadwaj, Bhatt, Vatsa and Richa Singh experiment with periocular regions including the eyebrows through GIST and CLBP. Robust LBP feature sets for periocular biometric identification is investigated by Juefei Xu etal. using Walsh masks, cosine transforms, LBP and Gabor filters [16]. Juefei-Xu et al. presented a frame work for age invariant face recognition using periocular region and gained $100 \%$ ranklidentification rate and 98\% verification rate [24]. Hollingsworth, Darnell, Miller, Woodard, Bowyer and Flynn have compared human and machine verification results of visible light and 
near-infra red light images. The experiments have obtained similar results of $88 \%$ accuracy for visible light images and 79\% for NIR images in both cases [26]. Juefei Xu and Savvides proposed an acquisition and recognition system based on periocular biometric using COTS PTZ camera to tackle difficulty posed in highly unconstrained environment. Periocular matching in all facial manners have achieved $60.7 \%$ verification rate with $0.1 \%$ false accept rate [27].

\section{Proposed System}

Periocular recognition system with reduced region of interest is proposed. The system relies on features extracted in LCPR. Chan-Vese active contour segmentation is performed to extract ROI. Triangulation method of partitioning these contours ensures natural edges of eye are embedded into the entropy of identifiers. The experiment is carried on two different sets of periocular data. Experiment 1 uses all the periocular features for the recognition purposes. Experiment 2 uses LCPR features alone for recognition. Bothe the experimentations consider direction of largest variance components using Principal Component Analysis (PCA). The results obtained from the two studies are compared.

\subsection{Pre-processing}

Images obtained from UBIRIS.v2 database are resized to $300 \mathrm{x} 400$. The RGB images are gray scale transformed. The gray scale function converts a 24-bit, three channel color image to an 8bit, single channel gray scale image by forming weighted sum of red, green and clue components. The texture variations of the skin in the periocular region are mapped to variations of intensity values. Histogram equalization is performed on resulting gray scale images. Equation 1 gives transformation for histogram equalization.

$$
S_{k}=T\left(r_{k}\right)=\sum_{j=0}^{k} p_{r}\left(r_{j}\right)=\sum_{j=0}^{k} \frac{n_{j}}{n} \quad k=0,1,2, \ldots \ldots L-1
$$

where $T$ is the transformation applied on input pixels $r_{k} \cdot P_{r}\left(r_{j}\right)$ is the probability of occurrence of pixel $r_{j}$, $n$ refers to the total number of pixels in the image and $n_{j}$ represents the number of pixels with $r_{j}$ intensity level.

\subsection{Region of Interest}

The size of periocular region to be cropped from images is an important consideration. Experiment 1 uses tight periocular areas around the eye. The ROI includes the upper/lower eyefolds and eye lids excluding the eye brows. To eliminate the effect of the iris and conjunctiva, an elliptical mask is placed on the eye. The pupil is detected using canny detector and is used as anchor point to mask. Only the skin texture information present in the periocular area is used for experimentation. Considering threats posed by artificial manipulation of texture information in ROI discussed in section 3, the strength of LCPR is examined in the second experiment. LCPR achieves good verification results comparable to the full periocular features with an advantage of fewer features. The region of interest is defined directly below eye edge and reaches up to start of cheek bone. The rich texture assures comparable results with those of experiment 1 . Intrusion of iris, sclera of eye and eyelashes is totally eliminated. In both the cases, the width of ROI is determined using end points of eye. These anchor points are detected using corner detection techniques. 


\subsection{Active Contour Segmentation}

Localization of iris is achieved using automatic segmentation software. Active contour segmentation technique developed by Chan and Vese is used [5]. The method does not require solutions of PDEs and are therefore fast. The method is able to automatically handle the topological changes [22]. This geometric active contour model begins with a contour in image plane defining an initial segmentation. The algorithm then evolves contour via a level set method in such a way that it stops on boundaries of foreground region as shown in figure 2 . In level set formulation, "mean curvature flow"- like evolving active contour stops on the desired boundary [17]. Level set function is defined as a value of gray level image at each pixel minus threshold. The asset of level set method is its ability to handle topological changes in edge contour, aiding to embed eye shape or contour information into periocular feature set.

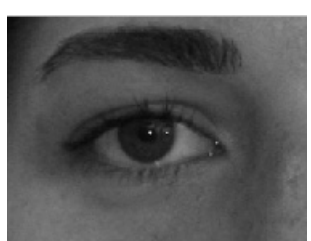

(a)

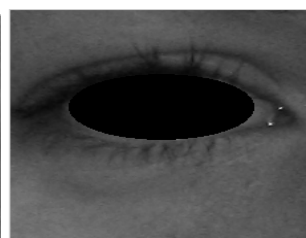

(b)

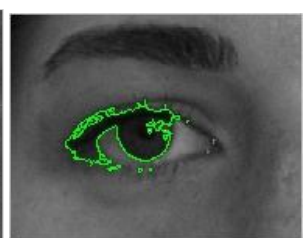

(c)

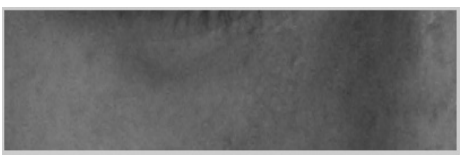

(d)

Figure 2. (a) Input image (b) Masked eye image (c) Segmentation using Active Contour methods (c) LCPR ROI

\subsection{Delaunay Triangulation}

Triangular segmentations of image plane have been proposed by Fisher [6]. The segmented periocular region is abstracted in terms of Delaunay triangulation as shown in figure 3. A Delaunay triangulation is a unique construction that no vertex from any triangle may lie within circum-circle of any other Delaunay triangle [7 and 23]. Satisfactory results are obtained using triangulation technique of partitioning in face recognition algorithms earlier [18]. The experiment uses image templates formed by pricking triangular meshes as graph nodes for periocular region authentication. Triangulation ensures extraction of geometric features of eye shape by preserving the topological features.

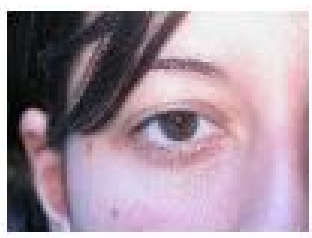

(a)

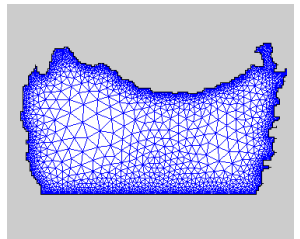

(b)

Figure 3. (a) Input image (b) Delaunay triangulation of corresponding periocular region

\subsection{Verification of periocular patterns using PCA}

Principal Component Analysis (PCA) is used for identifying patterns in data. PCA is informationpreserving statistical method useful for dimensionality reduction and feature extraction [19].

PCA projects data along directions where data varies most. These directions are determined by eigenvectors of covariance matrix corresponding to largest eigenvalues. Direction of Eigen 
vectors provides useful information of our data by extracting lines that characterize data. The lines pass through middle of data such that it draws a line of best fit. The eigenvector with highest eigenvalue is the principle components of data set [21].

\subsubsection{Computation of Eigen Periocular Images}

Periocular images in training set are represented as vectors. The average value of $M$ training images is calculated using equation 2 .

$$
\psi=\frac{1}{M} \sum_{n=1}^{M} \mathbb{\Gamma}_{n}
$$

Equation 3 determines difference of each training image from the mean image.

$$
\emptyset_{i}=\mathbb{T}_{i}-\psi
$$

Eigen vectors and eigenvalues are computed for the covariance matrix given by equation 4.

$$
C=\frac{1}{M} \sum_{n=1}^{M} \emptyset_{n}^{T} \emptyset_{n}
$$

Components of eigenvalues are arranged in order of significance from highest to lowest. Ordering eigenvectors in descending eigenvalues creates an ordered orthogonal basis with first eigenvector having direction of largest variance of data. Therefore, first set of Eigen images store class-specific information [16]. The eigenvalues are selected such that a maximum value given by equation 5 .

$$
\lambda_{k}=\frac{1}{M} \sum_{n=1}^{M}\left(u_{k}^{T} \emptyset_{n}\right)^{2}
$$

where $u_{l}^{T} u_{k}=\delta_{l k}=1$ if $l=k$ and 0 otherwise. The vectors $u_{k}$ are referred to as "Eigen periocular images". Eigen periocular images obtained for a sub set of experimental data is shown in figure 4.
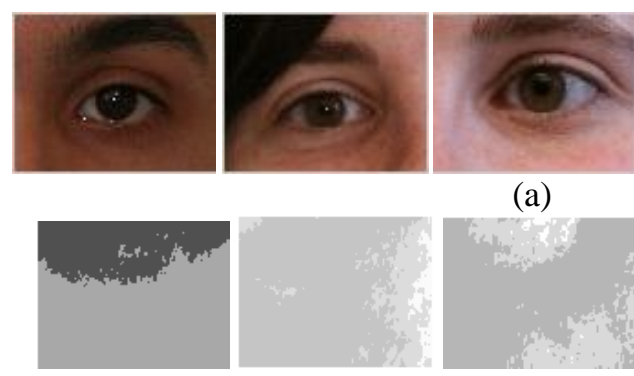

(a)

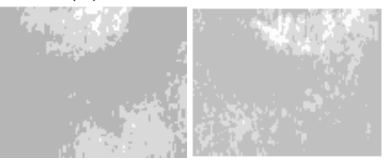

(b)
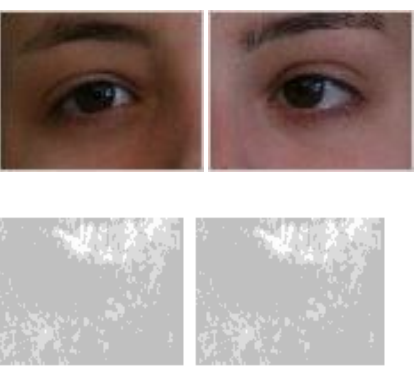

Figure 4. (a) Set of Original images (b) Corresponding periocular Eigen images 
Figure 5 shows the periocular Eigen images considering all the periocular features.

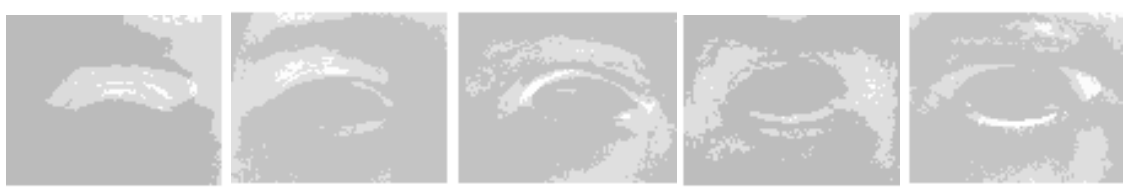

Figure 5. Periocular Eigen images with the elliptical mask

\subsubsection{Verification using Minimum distance classifier}

Minimum distance classifier using Euclidean distance is used to classify a test periocular image. It minimizes distance between image data and class in multi-feature space. The distance is defined as an index of similarity. Let $\mathbf{x}$ be feature vector for unknown input, and let $\mathrm{m}_{1}, \mathrm{~m}_{2} \ldots \mathrm{mc}$ be templates. Then, error in matching $\mathbf{x}$ against $m_{k}$ is given by $\left\|\mathbf{x}-m_{k}\right\|$. The minimum distance classifier computes this equation for $\mathrm{k}=1$ to $\mathrm{c}$ and chooses class for which distance is minimum.

\section{RESUlTS}

The scope of utility of UBIRIS.v2 database involves performance evaluation in the design of automatic authentication systems in civil and commercial applications. The major purpose of database is to constitute a new tool to evaluate feasibility of recognition under far from ideal imaging conditions $[20,25] .261$ subjects were imaged on-the-move, at-a-distance in visible wave length using Canon EOS 5D. 54.4\% were male and 45.6\% were female among 11102 images. Several challenges in scale, occlusion and illumination are exhibited. Occlusions included in images are flapped eyelids, eyelashes, hair and spectacles. Imaging was conducted at variable lighting conditions to emulate unconstrained environment.

A subset of periocular images from UBIRIS.v2 database which include variations in illumination and pose are used for both the experimentation. Experiment 1 and 2 were carried out using the same subset of images. Images with blinking are not considered. The subjects with spectacles are omitted. The images are first segmented and then triangulated to employ the natural edges of the eye, along with periocular texture for authentication. The number of training samples $\mathrm{M}=10$ was considered. The characteristic features in periocular images are extracted into Eigen periocular images using PCA mathematical tool.

For experiment 1, using the pupil as the anchor point for every image, an elliptical mask is placed on the eye in a way that the iris and the vascular conjunctiva do not bias the result. A tight periocular region excluding the eyebrows was considered. The experiment used the large amount of texture information present around the eye. The performance of the system is evaluated using standard biometric measures. The system achieved an acceptance rate of $100 \%$. The False Rejection Rate (Type I Error) and False Acceptance Rate (Type II Error) is not witnessed. The results obtained convince that the periocular region chosen achieves excellent biometric system.

Experiment 2 considered fewer features than those of experiment 1 . It uses only the LCPR region against the full periocular region as explored in the first experimentation. It achieves an acceptance rate of 90\%. False Rejection Rate (Type I Error) of 5\% and False Acceptance Rate (Type II Error) of 5\% results into total Rejection rate of 10\% is witnessed. The results obtained are comparable to those of full periocular features. Hence the proposed system uses reduced features of LCPR to obtain the accuracy nearing to those using more periocular entropy. 


\section{Conclusions}

The discriminative powers of LCPR in a periocular biometric system are investigated. The characteristics of the region are extracted using active contour segmentation and Delaunay triangulation in a way to imbibe the eye contour into the entropy of periocular. The proposed technique uses reduced region of interest and achieves False Rejection Rate of 5\%, False Acceptance Rate of 5\% using UBIRIS.v2 database through PCA mathematical tool against 100\% acceptance rate of entire periocular region.

\section{ACKNOWLEDGEMENTS}

This research uses UBIRIS.v2 database facilitated by SOCIA Lab, Soft Computing and Image Analysis Group, Department of Computer Science, University of Beira Interior, 6201-001, Covilha, Portugal.

\section{REFERENCES}

[1] Unsang Park, Jillela, Ross, Anil. K. Jain, (2011) "Periocular Biomtrics in the Visible Spectrum" IEEE Transactions on Information Forensics and Security, Vol. 6, No. 1.

[2] U. Park, A. Ross and A K Jain, (2009) "Periocular biometrics in the visibility spectrum: A feasibility study", proc. Biometrics: Theory, Applications and Systems (BTAS), pp.153 - 158.

[3] D.Woodard, S. Pundlik, P. Miller, Jamie R. Lyle,(2011), "Appearance - bsed periocular features in the context of face and non-ideal iris recognition", SIViP 5:443 - 455.

[4] P.Miller, A Rawls, S. Pundlik and D. Woodard, (2010), "Personal identification using Periocular skin texture", proc. ACM $25^{\text {th }}$ symposium on Applied Computing (SAC2010), pages 1496-1500.

[5] T.Chan and L.Vese, (2001), "Active contour without edges", IEEE transactions on image processing 10(2), pp. 266-277.

[6] Y.Fisher, Ed. (1995), "Fractal Image Compression - Theory and Application", Springer-Verlag.

[7] A.Bowyer (1981), "Computing Dirichlet Tessellations", Computer Journal, Vol.24, No. 2, 162-172.

[8] Jameson Merkow, Brendan Jou, Marios Savvides, (2010), “An Exploration of Gender Identification using only the periocular region", IEEE Transactions.

[9] Karen Hollingsworth, Kevin W.Bowyer and Patrick J.Flynn, (2010), "Identifying useful features for recognition in Near Infrared Periocular Images", IEEE Transactions

[10] Jamie. R.Lyle, P.Miller, S.Pundlik, D. Woodard, (2010), "Soft Biometric Classification using Peirocular Region Features", IEEE Transactions

[11] D.Woodard, S.Pundlik, Jamie.R.Lyle, P.Miller, (2010), "Periocular Region Appearance Cues for Biometric Identification", IEEE Transactions.

[12] Samarth Bharadwaj, Himanshu.S.Bhatt, Mayank Vatsa and Richa Singh, (2010), "Periocular Biometics: When Iris Recognition Fails", IEEE Transactions.

[13] Juefei Xu, Miriam Cha, Joseph L.Heyman, Shreyas Venugopalan, Ramzi Abiantum and Marios Savvides, (2010), "Robust local Binary Pattern Feature sets for Periocular Biometric Identification", IEEE Transactions.

[14] D.L.Woodard, S.Pundlik, P.Miller, R.Jillela and A.Ross, (2010), "On the fusion of periocular and iris biometrics in non-ideal imagery”, Proc. Int. Conf. on Pattern Recognition.

[15] J.Adams, D.L.Woodard, G.Dozier, P.Miller, K.Bryant and G.Glenn, (2010), "Genetic based Type II feature extraction for periocular Biometric Recognition: Less is more", Proc. Int. Conf. on Pattern Recognition.

[16] John Y Chiang, R.C.Wang, Yun-Lung Chang, "The Application of Triangulation to Face Recognition".

[17] Wenyi Zhao, Arvindh, Rama, Daniel, John, (1998), "Discriminant Analysis of Principal components for face recognition" Automatic Face and Gesture Recognition, Proc. Third International Conference

[18] Hugo Proenca, Silvio Filipe, Ricardo Santos, Joao Oliveira and Luis A.Alexandre, (2009), "The UBIRIS.v2: A Database of Visioble Wavelength Iris Images Captured On-The-Move and At-A Distance”, IEEE Transactions on pattern Analysis and Machine Intelligence. 
[19] Koray, Volkan, (2002), "PCA for gender estimation: Which Eigen Vectors contribute?", Pattern Recognition, $16^{\text {th }}$ International conference.

[20] Yongsheng Pan, Douglas, Seddik, (2006), "Efficient Implementation of the Chan Vese Models without solving PDEs", Multimedia Signal Processing, pages 350-354.

[21] Bebis.G, Deaconu, (1999) "Finger Print Identification using Delaunay triangulation", IEEE conference publication, Information Intelligence and Systems.

[22] Juefei Xu, Luu, Savvides, Bui, Suen, (2011), "Investigating age invariant face recognition based on periocular biometrics", IEEE conference publication, Biometrics (IJCB).

[23] Padole, Proenca, (2012), "Periocular recognition: Analysis of performance degradation factors", IEEE conference publication, Biometrics (ICB), $5^{\text {th }}$ IAPR International conference on Biometrics (ICB).

[24] Hollingsworth, Damell, Miller, Woodard, Bowyer, Flynn, (2012), "Human and Machine performance on Periocular Biometrics under Near-Infra red light and visible light", IEEE transactions on information forensics and security.

[25] Juefei Xu, Savvides, (2012), "Unconstrained periocular biometric acquisition and recognition using COTS PTZ camera for uncooperative and non-cooperative subjects", Applications of Computer Vision (WACV), IEEE.

\section{Authors}

Ambika D R is currently working for the department of Electronics and Communication Engineering, B.M.S College of Engineering. She received her B.E, M.E from Visvesvaraya Technological University, Belgaum. She is currently pursuing her $\mathrm{Ph}$. D in Biometric Authentication System.

Dr. Radhika K.R. joined department of Information Science and Engineering, B.M.S. College of Engineering in 1995, where she currently is the Professor. She received her B.E, M.E from Bangalore University and $\mathrm{PhD}$ from Visvesvaraya Technological University, Belgaum. Her research interests are Online and Offline Biometric Behavioral Pattern Authentication System using handwritten signature as a pattern. She is currently guiding three $\mathrm{PhD}$ students in the specialized areas of Biometrics. She is professional member of IEEE.

Dr. D Seshachalam is currently working as the Head of the Department, Electronics and Communication, B.M.S College of Engineering, Bangalore. He is awarded PhD Degree from MNNIT. His field of interests are Artificial Intelligence Control and Wireless Communication.
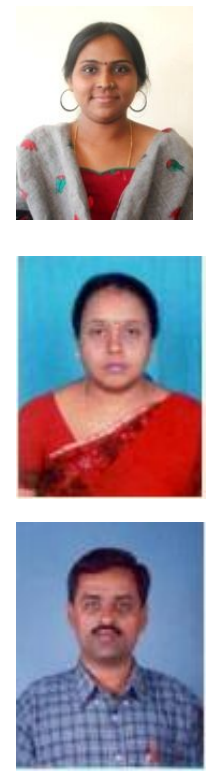